\title{
Struma ovarii: management and follow-up of a rare ovarian tumour
}

\author{
Jonathan Yeow Sherng $\underline{\text { Wee }}{ }^{1}$, MBBS, MRCoG, Xinyi $\underline{\mathrm{Li}}^{1}$, MBBS, Bernard Su Min $\underline{\mathrm{Chern}}{ }^{1}$, MBBS, FRCOG,
} Irene Sze Yuen Chua $^{1}$, MBBS, MRCOG

INTRODUCTION Struma ovarii represents about $1.0 \%$ of all ovarian tumours. While management involves surgery, there is a paucity of data regarding the extent and approach of surgery, and postoperative management. This study aimed to delineate the management of struma ovarii, its associated complications, and postoperative follow-up and investigations. METHODS We retrospectively reviewed cases of benign struma ovarii treated at KK Women's and Children's Hospital, Singapore, between January 2000 and May 2011.

RESULTS A total of 68 patients underwent surgical removal of ovarian cyst or mass (24 cystectomy, 20 salpingooopherectomy and 24 total hysterectomy and bilateral salpingo-oopherectomy). Of the 68 surgeries, 39 (57.4\%) included intraoperative frozen section sampling or procedures for staging of ovarian malignancy. The majority (73.5\%) of surgeries were laparotomies. Histology revealed benign struma ovarii in all (98.5\%) but one patient. Only $7(10.3 \%)$ patients had postoperative complications - 3 wound-related, 2 thyroid-related, 1 incisional hernia and 1 nonspecific. The mean length of hospital stay was 4.2 days. During follow-up, $45(66.2 \%)$ patients required no additional investigations. The most common investigation done was ultrasonography $(n=18,26.5 \%)$. While no recurrences were diagnosed histologically, two patients were subsequently found to have complex/dermoid ovarian cysts on the ipsilateral side of the previous struma ovarii on ultrasonography.

CONCLUSION Simple surgery is recommended for patients with struma ovarii, especially if they have fertility potential. Laparoscopic surgery is the recommended approach due to its shorter recovery time and lower morbidity. Most patients do not require extended periods of follow-up or postoperative investigations.

Keywords: monodermal ovarian teratoma, struma ovarii

\section{INTRODUCTION}

Struma ovarii is a rare ovarian tumour that has been reported to represent $0.5 \%-1.0 \%$ of all ovarian tumours. ${ }^{(1)}$ First described by Von Klden in 1895 and Gottschalk in 1899, struma ovarii is the most common type of monodermal teratoma, and comprises about $3.0 \%$ of all ovarian teratomas. ${ }^{(1-6)}$ It is defined as an ovarian teratoma that is composed predominantly of thyroid tissue (> 50\%), or forms a macroscopically recognisable component of mature cystic teratoma.

Historically, struma ovarii is managed via surgical removal of the ovarian cyst or mass, to enable a histological diagnosis to be made and to rule out ovarian malignancy. ${ }^{(1-4)}$ However, there is controversy regarding whether radical or conservative surgery should be practised, and what the optimum approach of surgical access should be. The aim of this study was to delineate the management of struma ovarii, its associated complications, and postoperative follow-up and investigations.

\section{METHODS}

A total of 68 cases of benign struma ovarii were diagnosed and treated at KK Women's and Children's Hospital (KKH), Singapore, between January 2000 and May 2011. The medical records of each case was retrospectively reviewed and analysed with respect to management and follow-up.
In all the cases, the management of struma ovarii was surgical removal. Data on the types of surgery, ranging from conservative surgeries (e.g. simple cystectomy, unilateral salpingo-oopherectomy) to radical surgery (e.g. total hysterectomy, with bilateral salpingo-oopherectomy [THBSO]), was reviewed. Details on the use of frozen section for intraoperative diagnosis and the employment of surgical staging techniques for ovarian malignancies (e.g. peritoneal washing, omentectomy, omental biopsy, appendectomy) were also recorded and analysed. In addition, surgical approaches (i.e. laparoscopic or open access) were studied and a temporal trend was obtained. We also analysed data on admission, length of hospital stay for surgery, complications of surgery, as well as length and number of sessions of postoperative follow-up and investigations.

\section{RESULTS}

All 68 patients in our cohort underwent surgical removal of the cyst or mass. Among these, 24 (35.3\%) patients underwent cystectomy, 20 (29.4\%) salpingo-oopherectomy and 24 (35.3\%) THBSO. None of the patients who indicated a desire for future fertility ( $n=15$ ) underwent radical surgery such as THBSO or bilateral salpingo-oopherectomy - $13(86.7 \%)$ underwent cystectomy and 2 (13.3\%) salpingo-oopherectomy (Table I).

${ }^{1}$ Department of Obstetrics and Gynaecology, KK Women's and Children's Hospital, Singapore

Correspondence: Dr Jonathan Yeow Sherng Wee, Consultant, Department of Obstetrics and Gynaecology, KK Women's and Children's Hospital, 100 Bukit Timah Road, Singapore 229899. jonthan@singnet.com.sg 
Table I. Surgical details of patients with struma ovarii $(n=68)$.

\begin{tabular}{lcc}
\hline Surgery performed & \multicolumn{2}{c}{ No. (\%) } \\
\cline { 2 - 3 } & $\begin{array}{c}\text { Patients who } \\
\text { desired conserved } \\
\text { fertility }(\mathbf{n}=\mathbf{1 5})\end{array}$ & $\begin{array}{c}\text { All } \\
\text { patients } \\
(\mathbf{n}=\mathbf{6 8})\end{array}$ \\
\hline Cystectomy & $13(86.7)$ & $24(35.3)$ \\
Salpingo-oopherectomy & $2(13.3)$ & $20(29.4)$ \\
Total hysterectomy and bilateral & $0(0)$ & $24(35.3)$ \\
salpingo-oopherectomy & & \\
\hline
\end{tabular}

A total of $39(57.4 \%)$ surgeries involved intraoperative frozen section sampling or procedures for surgical staging of ovarian malignancy (e.g. peritoneal washing, appendectomy, omentectomy, omental biopsy, macroscopic survey of intraabdominal organs, either individually or in combination), while 29 (42.6\%) patients did not undergo any additional procedure other than the primary surgery. Based on preoperative diagnoses from ultrasonography, a further breakdown revealed additional procedures in the following patients: 13 of the 20 (65.0\%) patients with a preoperative diagnosis of possible ovarian malignancy; 7 of the 13 (53.8\%) patients with a preoperative diagnosis of dermoid cyst or ovarian teratoma; and 5 of the $11(45.5 \%)$ patients with a preoperative diagnosis of endometriotic or haemorrhagic cyst.

An open approach to surgery was employed in the majority of cases $(n=50,73.5 \%)$, while a laparoscopic approach was used in the remaining 18 (26.5\%) cases. Interestingly, no surgery was performed via laparoscopy in 2000-2001 and 2003-2004. In 2002, 3 (16.7\%) surgeries were performed via laparoscopy. Between 2006 and 2010, laparoscopy was the method used in the majority of cases $(n=14,42.4 \%)$, with the greatest number of laparoscopic surgeries $(n=5,35.7 \%)$ performed in 2010. With regard to the open approach to surgery, the absolute number of such surgeries performed each year revealed no significant trend. However, when the percentage of open approach surgeries was compared with that of laparoscopic surgeries, we found that the former accounted for $88.6 \%$ (31 out of 35) of the surgeries performed between 2000 and 2005. However, the percentage of open approach surgeries dropped to 57.6\% (19 out of 33) from 2006 to 2010 (Fig. 1). Comparison of trend between laparoscopic and open approach surgeries using Fisher's exact test showed a statistically significant increase $(p=0.006)$ in the use of the laparoscopic approach between the periods 2000-2005 and 2006-2010 (Fig. 1).

Bilateral struma ovarii was not observed in any of the 68 patients. The size of the mass found during surgery ranged from $7 \mathrm{~mm} \times 6 \mathrm{~mm} \times 6 \mathrm{~mm}$ to $195 \mathrm{~mm} \times 135 \mathrm{~mm} \times 115 \mathrm{~mm}$. Histology revealed benign struma ovarii in all (98.5\%) but one case. In this single case of malignant struma ovarii, the frozen section of the right ovary obtained intraoperatively suggested a provisional benign diagnosis, and thus, a cystectomy was performed. However, histopathology subsequently revealed microscopic vascular invasion and the presence of irregular tongue-like projections into the surrounding tissue, which led to the histological diagnosis of malignant struma ovarii. After discussion with the tumour board, it was decided that the patient

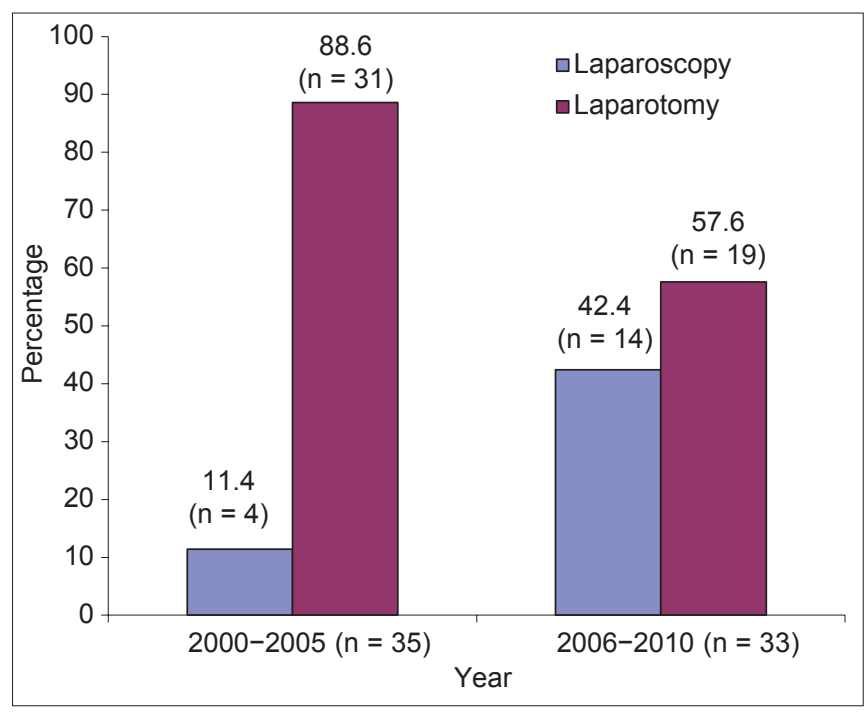

Fig. 1 Trend of laparotomy vs. laparoscopic surgery approaches from 2000 to 2010. $p=0.006$ for comparison of percentage of laparoscopic approach in struma ovarii surgery between the periods 2000-2005 and 2006-2010.

should not undergo further invasive management. Instead, the patient was scheduled for yearly follow-up, during which ultrasonography was performed and CA125 values recorded. All subsequent follow-up and investigations did not suggest any recurrence of the tumour, or metastasis. Therefore, the patient was eventually discharged after a total of 76 months of follow-up.

Among the 68 patients, 7 (10.3\%) had post-surgery complications - $3(42.9 \%)$ had wound breakdown, inflammation or infection; 2 (28.6\%) developed thyroid-related problems; $1(14.3 \%)$ suffered from incisional hernia one year after surgery; and $1(14.3 \%)$ was readmitted after discharge due to shortness of breath and diarrhoea. Three of the four patients who suffered wound-related complications after surgery were above 50 years old, and another three of the four had multiple comorbidities, including diabetes mellitus and morbid obesity. All patients with wound-related complications were successfully treated with antibiotics and secondary sutures, and achieved full recovery. Of the two patients who developed thyroid-related problems after surgery, one developed hyperthyroidism four years after surgery and was treated with propylthiouracil, while the other patient's information was unavailable. It would have been ideal if details on both patients' thyroid problems were available, as this would have enabled us to evaluate the possible correlation between the removal of struma ovarii and the subsequent development of thyroid-related problems. However, such information was unavailable at the time of data collection and analysis. The single patient with incisional hernia underwent an uneventful hernia repair. The patient who was admitted a few days after discharge with complaints of shortness of breath and diarrhoea was treated symptomatically after pulmonary embolism was ruled out, and made full recovery.

The length of inpatient stay ranged from 0 to 37 days, with a mean length of stay of 4.2 days. Most of the patients $(n=56$, $82.4 \%$ ) had a hospital stay of $\leq 4$ days for their surgery. This included $77.6 \%$ of patients who underwent laparotomy and $94.7 \%$ who underwent laparoscopy (Table II). Most of the patients 
(95.6\%) were admitted either on the same day or one day before their scheduled date of surgery.

With regard to postoperative follow-up, the total number of follow-up sessions after hospital discharge ranged from 0 to 13. One patient had no data regarding follow-up. The mean number of follow-up sessions was 2.7, with 51 (76.1\%) patients having $\leq 3$ follow-up sessions after surgery. The duration of postoperative follow-up (i.e. the length of time after surgery to the time of discharge/default from follow-up) ranged from 0 to 76 months, and the mean duration of follow-up was 12.5 months. This information did not include data for five patients, as these patients were either unavailable or still on follow-up for struma ovarii. The duration of postoperative follow-up was $\leq 1$ month for $21(30.9 \%)$ patients, > 1-12 months for 22 (32.4\%) patients and > 1 year for 20 (29.4\%) patients (Table III).

During postoperative follow-up, more than half of the patients ( $n=45,66.2 \%$ ) did not have additional investigations performed other than clinical history-taking and physical examination. The most commonly performed investigation was ultrasonography of the pelvis ( $\mathrm{n}=18,26.5 \%$ ), followed by thyroid function test $(\mathrm{n}=8,11.8 \%)$, CA125 test $(\mathrm{n}=3,4.4 \%)$, computed tomography $(\mathrm{n}=2,2.9 \%)$ and ultrasonography of the thyroid $(\mathrm{n}=1,1.3 \%)$. Among the patients who underwent ultrasonography of the pelvis after surgery, the images of 15 (83.3\%) patients did not reveal any recurrence of ovarian cyst or mass, while that of $2(11.1 \%)$ patients showed recurrence of a functional cyst on the same side as the resected struma ovarii and that of $1(5.6 \%)$ patient revealed a haemorrhagic cyst on the contralateral side of the previously resected struma ovarii.

While all other postoperative investigations done within the duration of the follow-up did not reveal any abnormal results that were suggestive of recurrence, one patient (who underwent postoperative ultrasonography of the thyroid) was found to have four thyroid nodules. This patient's thyroid function test was normal and she was clinically asymptomatic. There was no follow-up for her thyroid nodules and she did not undergo any treatment for this condition in $\mathrm{KKH}$.

No recurrence of struma ovarii was histologically diagnosed in any of the 68 patients during their respective duration of follow-up. Two patients were, however, found to have ovarian cysts on the ipsilateral side of their previous struma ovarii on ultrasonography. The cysts of these two patients were detected on ultrasonography 2 and 4.5 years, respectively, after they were discharged from outpatient follow-up. A differential diagnosis of dermoid cyst or ovarian teratoma was offered in one patient, while the other patient's report suggested a complex cyst or solid lesion with no specific differential. As neither patient underwent surgical removal of the cyst (as they were not keen to undergo surgery), we were unable to obtain histological diagnoses of the cysts to confirm or exclude recurrence. However, both of these patients remained clinically asymptomatic.

\section{DISCUSSION}

As described in previous reports, the key management of struma ovarii is surgical removal. ${ }^{(1-4)}$ In the present study, 44 (64.7\%)
Table II. Length of inpatient stay based on the surgical approach used $(n=68)$.

\begin{tabular}{lcc}
\hline \multirow{2}{*}{$\begin{array}{l}\text { Length of inpatient } \\
\text { stay (day) }\end{array}$} & \multicolumn{2}{c}{ No. (\%) } \\
\cline { 2 - 3 } & Laparotomy & Laparoscopy \\
\hline$\leq 4$ & $38(77.6)$ & $18(94.7)$ \\
$>4$ & $11(22.4)$ & $1(5.3)$ \\
Total & $49(100.0)$ & $19(100.0)$ \\
\hline
\end{tabular}

Table III. Duration of postoperative follow-up of the patients with struma ovarii $(n=68)$.

\begin{tabular}{lc}
\hline Duration of postoperative follow-up (mth) & No. (\%) \\
\hline$\leq 1$ & $21(30.9)$ \\
$>1-12$ & $22(32.4)$ \\
$>12$ & $20(29.4)$ \\
Not applicable & $5(7.4)$ \\
\hline
\end{tabular}

patients underwent fertility-conserving surgery (includes cystectomy and unilateral salpingo-oopherectomy), regardless of their stated desire or potential for fertility. None of the patients who stated a desire for future fertility underwent radical surgery. This is in concordance with Ayhan et al's study, ${ }^{(7)}$ which recommended fertility-conserving management for those with benign struma ovarii, and radical staging surgery for patients who have malignant struma ovarii and no future fertility desire.

As only one patient in the present study had malignant struma ovarii, we were unable to comment on the management of malignant struma ovarii. However, it is noteworthy that the use of conservative surgery and no adjuvant therapy for the one case of malignant struma ovarii, which was of low-grade malignancy and confined to the ovary, did not show signs of recurrence or metastasis for up to seven years after surgery. This finding supports previous studies that suggest that struma ovarii is of low malignancy and low metastatic potential. ${ }^{(4,7,8)}$ A study by DeSimone et al also supports the consideration of conservative surgery for women with malignant struma ovarii who had childbearing potential, ${ }^{(8)}$ although the authors recommended the use of radioactive iodine adjuvant therapy to reduce the rates of recurrence. ${ }^{(8)}$ The use of iodine-131 as adjuvant therapy in malignant struma ovarii remains controversial due to the paucity of information and the lack of large prospective trials; thus far, the results seem to be dependent on the individual patient and primary team. ${ }^{(8,9)}$

In the present study, just over half of the study cohort underwent frozen section sampling or staging procedures as part of their surgery. These procedures included omental biopsy, omentectomy, appendectomy and peritoneal washing, either in combination or alone. The percentage of patients who underwent additional frozen section sampling or staging surgery varied according to their preoperative ultrasonography diagnoses. Patients who had malignancy as the preoperative diagnosis made up the highest percentage of patients who underwent additional frozen section sampling or staging surgery, followed by those with dermoid cyst or ovarian teratoma, and finally, those with endometriotic or haemorrhagic cysts. However, it should be 
noted that multiple preoperative diagnoses may be offered within a single ultrasonography report, and this may dilute the strength of direct correlation between the preoperative diagnosis and the actual surgery performed.

In contrast, Yoo et al's study reported that infracolic omentectomy was performed for only two patients diagnosed with malignant struma ovarii. ${ }^{(1)}$ There are a few possible explanations for the difference between our findings and that of Yoo et al's study. Firstly, many cases of struma ovarii in the present study were preoperatively diagnosed as possible malignancies based on the findings of the investigations. Hence, it was fully expected that planned radical surgeries for these patients would include frozen sections and staging procedures. However, intraoperatively, these surgeries would have been converted to more conservative procedures, if the findings on frozen section indicated the absence of malignancy. Therefore, this resulted in a large proportion of conservative surgeries being associated with staging procedures. Secondly, as struma ovarii is a rare tumour with a relatively nonspecific nature of presentation, which often overlaps and mimics ovarian malignancy, ${ }^{(3,10,11)}$ many surgeons may not have considered it a likely differential intraoperatively. Therefore, additional procedures would have been carried out before histological diagnosis was obtained based on frozen section sampling.

As the present study spanned more than a decade, we were able to describe trends in the approach to surgery (i.e. laparotomy or laparoscopy) for struma ovarii management. Even though the number of surgeries performed via open access exceeded the number of surgeries via laparoscopy, our results showed a clear trend of increasing popularity of laparoscopic surgery. This was especially eminent in the recent five years - a statistically significant increase in the use of laparoscopic access was observed when the approach to surgery used in 2000-2005 was compared with that used in 2006-2010 ( $p=0.006)$. Moreover, in the present study, none of the surgeries performed via laparoscopy required conversion to laparotomy.

Access via laparoscopy for the removal of large ovarian cysts is now well established and technically feasible for struma ovarii. ${ }^{(12-15)}$ As struma ovarii is often a histological diagnosis that is clinched postoperatively, there is concern that the removal of a potentially malignant ovarian mass via laparoscopy may decrease the accuracy of staging, and increase the possibility of port site metastasis and spillage of cancerous cells into the abdominal cavity. However, a review by Ezon et al reported that the use of the laparoscopic approach did not negatively impact disease-free or overall survival rates. ${ }^{(12)}$ In fact, no case of port site metastasis was noted and spillage of tumour content during surgical resection was not found to negatively impact patient survival. ${ }^{(12)}$

In the present study, only $1(1.5 \%)$ patient was diagnosed with malignant struma ovarii. This patient underwent surgery via laparotomy, with no complications or recurrence observed during the 76 months of follow-up. Patients who underwent laparoscopic surgery did not suffer any intraoperative complications, and none required conversion to laparotomy. Moreover, these patients also had a shorter length of inpatient stay (mean length of inpatient stay $=1.9$ days, mode $=1$ day) than the patients who underwent laparotomy (mean length of inpatient stay $=5.1$ days, mode $=5$ days). Furthermore, all the patients who experienced postoperative complications (including wound inflammation, infection and subsequent incisional hernia) in the present study were those who had undergone surgery via laparotomy. In the present study, it is evident that laparoscopic surgery resulted in shorter length of hospital stay and lower morbidity as compared to laparotomy. These findings are similar to those found in a review conducted by Medeiros et al, which examined the advantages and disadvantages of laparotomy and laparoscopy for benign ovarian tumours. ${ }^{(16)}$

To the best of our knowledge, no other study has reported results with regard to the length of hospital stay among patients with struma ovarii. In the present study, most of the patients stayed in the hospital for a maximum of four days, after which they were discharged home well without incident. Most were also admitted either on the same day as their scheduled surgery or one day prior to surgery. This information can be used as a guide to advise future patients who are scheduled to undergo surgery for struma ovarii or patients who are recovering from similar surgeries.

In the present study, the mean number of follow-up sessions conducted after surgery was 2.7 ; approximately $75 \%$ of the patients had three or less follow-up sessions. About one-third of the patients had a total duration of follow-up of one month or less, while another one-third had a total duration of follow-up of more than one month to a year. The remaining one-third of the patients, including one patient with malignant struma ovarii, had more than one year of follow-up. The patient with malignant struma ovarii had the longest duration of follow-up (i.e. 76 months). Moreover, regular follow-up sessions were planned for patients even if their conditions were benign in nature. This contrasts with the practice reported by Yoo et al, in which only patients with malignant struma ovarii had regular follow-up for a median of 65 months. ${ }^{(1)}$

Although postoperative follow-up was planned for almost all the patients in the present study, not every patient underwent postoperative investigations. About two-thirds of the patients did not undergo any form of postoperative investigation. The most commonly performed investigation was ultrasonography of the pelvis. Thyroid function tests were ordered for eight patients, one of whom was the patient diagnosed with malignant struma ovarii. This patient, who did not undergo any thyroid function test before diagnosis, had two sets of normal results after surgery. Of the remaining seven patients who underwent thyroid function tests after surgery, only one was done preoperatively (both the pre- and postoperative tests yielded normal results). However, the indication for thyroid function test before surgery was not explicitly stated in the medical records, and the patient was asymptomatic and had no past medical history. The postoperative thyroid function tests of the other six patients were normal, and they remained clinically asymptomatic for both hyper- and hypothyroidism. In view of the diagnoses, it is likely that the indication for these postoperative thyroid function tests was to establish thyroid status, as previous reports have suggested the possibility of hypothyroidism after surgical removal of struma ovarii. ${ }^{(12,17)}$ However, unless the patients 
are symptomatic for hypothyroidism, we do not recommend that postoperative thyroid function tests be performed for every patient diagnosed with struma ovarii.

In the present study, two patients were suspected to have recurrent ovarian cyst on the ipsilateral side of their previous struma ovarii. However, as neither patient consented to the surgical removal of the cysts, no histological diagnosis could be obtained to ascertain if the cysts were indeed a recurrence of struma ovarii. Data on recurrence of benign struma ovarii is scarce, while that on malignant struma ovarii is relatively low. ${ }^{(1,7,11)}$ Of note, among the two patients with recurrent cysts, one underwent removal of struma ovarii via laparoscopy, while the other via laparotomy. This finding does not concur with the conclusions made by Laberge and Levesque, who suggested that surgery via laparoscopy has a higher risk of recurrence. ${ }^{(18)}$

In conclusion, conservative surgery is recommended for patients who have benign struma ovarii, especially if they have potential for fertility and desire fertility. In addition, laparoscopic surgery is recommended as the surgical approach for the removal of struma ovarii, as it results in a shorter length of hospital stay and a lower incidence of postoperative complications when compared to laparotomy. As evidenced in the present study, most of the patients who underwent laparoscopic surgery recovered without incident, had a short duration of hospital stay and did not require extended periods of follow-up or excessive postoperative investigations unless symptomatic.

\section{REFERENCES}

1. Yoo SC, Chang KH, Lyu MO, et al. Clinical characteristics of struma ovarii. J Gynecol Oncol 2008; 19:135-8.

2. Lochrane CD. Struma Ovarii. Proc R Soc Med 1933; 26:1427-9.
3. Mancuso A, Triolo O, Leonardi I, De Vivo A. Struma ovarii: a rare benign pathology which may erroneously suggest malignancy. Acta Obstet Gynecol Scand 2001; 80:1075-6.

4. Raina A, Stasi G, Monzio Compagnoni B, et al. Struma ovari--a rare gynecological tumor. Acta Oncol 1997; 36:533-4

5. Jacobsen GK, Talerman A, eds. Atlas of Germ Cell Tumours. Copenhagen: Munksgaard, 1989.

6. Scully RE, Young RH, Clement PB. Tumors of the Ovary, Maldeveloped Gonads, Fallopian Tube, and Broad Ligament. In: Atlas of Tumor Pathology. 3rd series, Fascicle 23. Washington DC: Armed Forces Institute of Pathology, 1992.

7. Ayhan A, Yanik F, Tuncer R, Tuncer ZS, Ruacan S. Struma ovarii. Int J Gynaecol Obstet 1993; 42:143-6.

8. DeSimone CP, Lele SM, Modesitt SC. Malignant struma ovarii: a case report and analysis of cases reported in the literature with focus on survival and I131 therapy. Gynecol Oncol 2003; 89:543-8.

9. Volpi E, Ferrero A, Nasi PG, Sismondi P. Malignant struma ovarii: a case report of laparoscopic management. Gynecol Oncol 2003; 90:191-4.

10. Szyfelbein WM, Young RH, Scully RE. Struma ovarii simulating ovarian tumors of other types. A report of 30 cases. Am J Surg Pathol 1995; 19:21-9.

11. Rim SY, Kim SM, Choi HS. Struma ovarii showing clinical characteristics of ovarian malignancy. Int J Gynecol Cancer 2005; 15:1156-9.

12. Ezon I, Zilbert N, Pinkney L, et al. A large struma ovarii tumor removed via laparoscopy in a 16-year-old adolescent. J Pediatr Surg 2007; 42:E19-22.

13. Shalev E, Bustan M, Romano S, Goldberg Y, Ben-Shlomo I. Laparoscopic resection of ovarian benign cystic teratomas: experience with 84 cases. Hum Reprod 1998; 13:1810-2.

14. Jeong EH, Kim HS, Ahn CS, Roh JS. Successful laparoscopic removal of huge ovarian cysts. J Am Assoc Gynecol Laparosc 1997; 4:609-14.

15. Canis M, Rabischong B, Houlle C, et al. Laparoscopic management of adnexal masses: a gold standard? Curr Opin Obstet Gynecol 2002; 14:423-8.

16. Medeiros LR, Rosa DD, Bozzetti MC, et al. Laparoscopy versus laparotomy for benign ovarian tumour. Cochrane Database Syst Rev 2009; 15:CD004751.

17. Carvalho JP, Carvalho FM, Lima de Oliveira FF, Asato de Camargo RY. Hypothyroidism following struma ovarii tumor resection: a case report. Rev Hosp Clin Fac Med Sao Paulo 2002; 57:112-4.

18. Laberge PY, Levesque S. Short-term morbidity and long-term recurrence rate of ovarian dermoid cysts treated by laparoscopy versus laparotomy. J Obstet Gynaecol Can 2006; 28:789-93. 